\title{
28 Resarch Square \\ Neuroendocrine Carcinoma of the Cervix: Clinicopathologic Study and Review of the Literature
}

\author{
Xiaobo Zhang \\ Peking University, People 's Hospital \\ Yougui $X u$ \\ Peking University, People 's Hospital \\ Danhua Shen ( $\nabla$ danhuashen@126.com ) \\ Peking University, People 's Hospital \\ MingZhu LI
}

\section{Research Article}

Keywords: Cervical neuroendocrine carcinoma, Small cell neuroendocrine carcinoma, pathology, Immunohistochemistry, Prognosis

Posted Date: November 11th, 2021

DOI: https://doi.org/10.21203/rs.3.rs-1033140/v1

License: @ (i) This work is licensed under a Creative Commons Attribution 4.0 International License. Read Full License 


\section{Abstract}

Background

Neuroendocrine carcinoma of the cervix (CNECC) is a rare variant of cervical cancer. The prognosis of women with CNECC is poor and there is no standardized therapy for this type of malignancy. To discuss the clinical and pathological features and prognosis of CNECC.

Methods

Twenty one patients diagnosed as CNECC of cervix from May 2008 to September 2021 were retrospectively analyzed at Peking University people's hospital, were analyzed retrospectively including hematoxylin-eosinstaining $\triangle$ HE囚slides review, immunohistochemistry results, Thinprep cytology test(TCT) and human papillomavirus (HPV) Hybrid Capture 2(HC2) assay. and their data were analyzed retrospectively. Telephone and medical records were followed up for 3-160 months with an average follow-up time of 49.8 month.

Results

The patient's average age was 48.6 years old (range: 33-69 years). The first symptoms of 11 cases had vaginal bleeding, 2 cases had vaginal discharge, and the others were asymptomatic. Among the 21 patients, 17 cases were diagnosed as neuroendocrine carcinoma by biopsy. There were 9 cases with TCT examination and HC2 tests before biopsy, TCT results of 4 cases were positive. High-risk HPV of 7 cases were positive. The morphology of cancer cells were relatively consistent, the cytoplasm was sparse, the nuclei were obviously blue stained, and accompanied by extensive neoplastic necrosis. 13 cases were pure CNECC(61.9\%), 8 cases were mix types of CNECC(38.1\%).There were 3 cases accompanied by squamous cell carcinoma, 5 cases accompanied by adenocarcinoma. The positive detection rate of Syn, CgA, CD56,p16 and TTF1 were 85.7\%(18/21), 42.9\%(9/21), 85.7\% (18/21), 81\%(17/21) 52.4\% (11/21),respectively. The overall survival rate of 21 NECC cases was $71.4 \%(15 / 21)$.

Conclusions

CNECC was a extremely rare primary tumor. The tumor was associated with HPV infection. Combined examination of TCT and HPV could significantly improve the detection rate of neuroendocrine carcinoma before biopsy.Pthology diagnosis was based on histological and immunohistochemical examination. It was considered to be highly aggressive malignancy with very poor prognosis.

\section{Introduction}

Cervical cancer was one of the most common malignant tumors of the female reproductive system, and the most common pathological type was squamous cell carcinoma, accounting for more than $90 \%(1)$ While neuroendocrine cancer was more common in the lungs, digestive tracts, other tissues and organs were rare, the primary cervical CNECC was more rare.CNECC was an aggressive histological variant of cervical cancers, only accounting for about $1-1.5 \%$ of all primary cervical cancers $(2,3)$. Its clinical manifestation was similar to the squamous cell carcinoma and adenocarcinoma of cervix.Early cervical neuroendocrine cancer was detected difficultly(4). But the biology of CNECC was different from squamous cell carcinoma or adenocarcinoma of the cervix regarding a number of characteristics. Compared with the latter, the former was characterized by high degree of malignancy, strong aggressiveness, poor prognosis.CNECC was more likely to invade the lymphvascular space and spread to the regional lymph nodes at the time of initial diagnosis(5).In this paper, the clinical manifestations, histopathological features, immunohistochemistry, etc,the clinic pathological features of CNECC were retrospectively analyzed, and the relevant literatures were reviewed, so as to highlight the pathological characteristics, diagnostic criteria and prognosis of women with CNECC,improve the understanding of this tumor and avoid missed and delayed diagnosis in clinical work.

\section{Material And Methods}

\subsection{Clinical data}

21 cases of CNECC treated in Peking University people's hospital from May 2008 to September 2021 were retrospectively analyzed.All cases were re-reviewed by two senior gynecological pathologists, and all cases were confirmed to be primary CNECC based on the general check-up, laboratory examination and follow-up. 


\subsection{Cytology examination}

Pretreatment cytologic slides were made using Thinprep cytology test (Hologic,Bedford,MA). All cytologic results were reported according to The Bethesda System (TBS) terminology.

\subsection{HPV test}

9 patients had high-risk HPV test using Hybrid Capture 2 assay (HC2, Digene, Gaithersburg, MD, USA). Greater than or equal to $1 \mathrm{pg} / \mathrm{ml}$ HPV DNA was identified as positive.

\subsection{Immunohistochemical(IHC)analysis}

All the pathologic specimens were fixed with $4 \%$ neutral formaldehyde, and the wax were routinely dehydrated and soaked, embedded in paraffin, and sectionalized by HE staining. All immunohistochemical tests were performed according to manufacturers' protocols.p53 (D07, Roche, Roche Benchmark Ultra), p16 (E6H4, Roche, Roche Benchmark Ultra), ER (6F11, Leica, RTU), PR (16, Leica, RTU), CK7 (EP16, zhongshanjinqiao, 1:100), CK20(EP23,zhongshanjinqiao, 1:100), CDX-2(EP25, zhongshanjinqiao, 1:200),Ki67(EP5,zhongshanjinqiao,1:200).Syn(UMAB112,zhongshanjinqiao,1:100),CgA(EP38, zhongshanjinqiao, 1:100), CKpan(AE1/AE3, zhongshanjinqiao, 1:200).Brown-yellow staining was considered as positive staining, and Ki-67, TTF1 and CDX2 positive sites were located in the nucleus. CKpan, CK7, CK20, Syn, CgA positive cells were located in the cytoplasm.

\section{Results}

\subsection{Clinicopathological features}

The study cohort included 21 patients diagnosed with the primary CNECC.The patient's average age was 48.6 years old (range: 33-69 years). The first symptoms were varied, among the 11 patients had vaginal bleeding, 2 patients had vaginal discharge, and the others were asymptomatic. Among the 21 patients, 17 cases were diagnosed as neuroendocrine carcinoma by biopsy and 4 cases were diagnosed as other tumors, and they were found in the radical resected specimens. None of the cases have received chemotherapy before diagnosis. 16 cases underwent extensive hysterectomy-bilateral adnexa-pelvic lymph node and/or para-aortic lymph node resection, and 1 patient underwent cervical conical resection. 5 patients received neoadjuvant chemotherapy before surgery, 12 patients received adjuvant chemotherapy after surgery.

\subsection{Cytological results}

9 cases had TCT examination before biopsy, 5 cases were negative, 4 cases were positive, and they were high-grade squamous intraepithelial lesion(HSIL)(table 1). Cytology were characterized by scattered or clumpy distribution of small cells, high cytoplasm, hyperchromatic nuclei, inconspicuous nucleoli, and common mitotic images and apoptotic bodies(Fig 1).

\subsection{HPV results}

9 cases had HPV tests, among of 7 cases were positive for high-risk HPV and 2 cases were negative(table 1).

\subsection{Pathological detection}

On gross examination,most cases were similar to other cervical carcinomas, showed exogenous polyps or cauliflower-like pattern. The maximum tumor diameter ranged from 2.5 to $7.0 \mathrm{~cm}$ in size. The cut surfaces were often white or gray-red, solid with hemorrhage and necrosis, crisp or hard(fig 2). Microscopically, among of 20 cases,tumor cells were usually in the form of adenoids, bands, clusters or diffuse patches, and the surface epithelium weren't invaded. The morphology of cancer cells were relatively consistent, the cytoplasm was sparse, the nuclei was obviously blue stained, and was accompanied by extensive neoplastic necrosis,and considered as small cell neuroendocrine carcinoma (SCNEC)(fig 3). 1 case of tumor cells were bigger relatively, organ-like structures maybe seen,and considered as large cell neuroendocrine carcinoma (LCNEC) (Fig 3).In this group, 13 cases were pure CNECC (61.9\%), in pure CNECC, two cases were accompanied by HSIL,one case was accompanied by AIS.8 cases were mix types of CNECC(38.1\%), coexistent with an adenocarcinoma, or squamous cell carcinoma. There were 3 cases accompanied by squamous cell carcinoma, 5 cases accompanied by adenocarcinoma冈table $1 \otimes . S y n, C g A$ and CD56 were positive in different degrees, and the positive rates were $85.7 \%(18 / 21), 42.9 \%$ (9/21), 85.7\% (18/21). The positive rate of epithelial markers ,CKpan, EMA and CEA were 90.5\% (19/21),61.9\% (13/21) and 38.1\% 
$(8 / 21)$ respectively. The positive rate of $p 16$ was $81 \%(17 / 21)$, The positive rate of p63 was $42.9 \%(9 / 21)$. The positive rate of TTF1 was $52.4 \%(11 / 21)$. The positive rate of CK7 was 100\%(21/21), The positive rate of CK20 was negative for all of 21 cases.

\section{Table 1}

Summary of clinicopathological features in the part of patients with CNECC

\begin{tabular}{|c|c|c|c|c|c|c|c|c|c|c|}
\hline $\begin{array}{l}\text { Patient } \\
\text { ID }\end{array}$ & Age(Years) & $\begin{array}{l}\text { Clinical } \\
\text { feature }\end{array}$ & TCT & HPV & Biopsy & $\begin{array}{l}\text { Tumor } \\
\text { size }(\mathrm{cm})\end{array}$ & $\begin{array}{l}\text { Pathologic } \\
\text { diagnosis }\end{array}$ & $\begin{array}{l}\text { Accompanying } \\
\text { diagnosis }\end{array}$ & Operation & Prognosis \\
\hline 1 & 49 & $\begin{array}{l}\text { vaginal } \\
\text { bleeding }\end{array}$ & $\mathrm{N}$ & $P$ & SCNEC & $3 \times 2.5 \times 2$ & SCNEC & No & EHBAPLN & alive \\
\hline 2 & 49 & $\begin{array}{l}\text { cervical } \\
\text { mass }\end{array}$ & $\mathrm{N}$ & $P$ & SCNEC & $2.5 \times 1.8$ & SCNEC & No & EHBAPLN & alive \\
\hline 3 & 49 & $\begin{array}{l}\text { cervical } \\
\text { mass }\end{array}$ & HSIL & $P$ & SCNEC & $1.8 \times 0.9$ & SCNEC & HSIL & EHBAPLN & alive \\
\hline 4 & 58 & $\begin{array}{l}\text { vaginal } \\
\text { bleeding }\end{array}$ & $\mathrm{N}$ & $\mathrm{N}$ & SCNEC & $1.5 \times 0.8$ & SCNEC & No & EHBAPLN & alive \\
\hline 5 & 58 & $\begin{array}{l}\text { cervical } \\
\text { mass }\end{array}$ & $\mathrm{N}$ & $\mathrm{N}$ & SCNEC & $2 \times 0.8$ & SCNEC & No & CCR & Lost \\
\hline 6 & 60 & $\begin{array}{l}\text { vaginal } \\
\text { bleeding }\end{array}$ & HSIL & $\mathrm{P}$ & HSIL & $0.8 \times 0.5$ & SCNEC & SCC & EHBAPLN & alive \\
\hline 7 & 45 & $\begin{array}{l}\text { cervical } \\
\text { mass }\end{array}$ & HSIL & $P$ & SCNEC & $2.5 \times 2$ & SCNEC & HSIL & EHBAPLN & death \\
\hline 8 & 49 & $\begin{array}{l}\text { cervical } \\
\text { mass }\end{array}$ & $\mathrm{N}$ & $\mathrm{P}$ & SCNEC & $2 \times 1$ & SCNEC & No & EHBAPLN & alive \\
\hline 9 & 62 & $\begin{array}{l}\text { vaginal } \\
\text { bleeding }\end{array}$ & HSIL & $P$ & SCNEC & $2 \times 2$ & SCNEC & SCC & EHBAPLN & alive \\
\hline
\end{tabular}

Note:N:negative;P:positive;EHBAPLN:extensive hysterectomy bilateral adnexa pelvic lymph node

CCR:cervical conical resection

\subsection{Follow-up}

Telephone and medical records were followed up for 3-160 months with an average follow-up time of 49.8 month. Of the 21 patients, 20 received surgery and 1 did not receive surgery. 6 patients died and 3 patients lost to follow-up. The remaining 12 patients were alive, no recurrence or metastasis.

\section{Discussion}

Cervical neuroendocrine tumor was a tumor derived from neuroendocrine cells. In 1957, The disease was first reported by Reagan et al(6).Primary neuroendocrine cervical tumor was extremely rare, accounting for only $1 \%$ to $1.5 \%$ of cervical primary tumors(2).In 1997 , the American society for cancer research divided CNECC into four types: typical carcinoid, atypical carcinoid, LCNEC and SCNEC(7).In the 2014 WHO classification of the female reproductive system tumor (1) recommended to reference the classification methold of the gastrointestinal tract, pancreatic neuroendocrine tumor, the cervix neuroendocrine tumors could be divided into low grade neuroendocrine tumor and high grade neuroendocrine carcinoma, low grade tumor including low grade neuroendocrine tumor grade 1 and grade 2, synonymous with its corresponding typical carcinoid and atypical carcinoid, respectively, high grade neuroendocrine carcinoma were divided into SCNEC and LCNEC, synonymous with its corresponding small cell and large cell carcinoma, respectively. While in the $2020 \mathrm{WHO}$ classification of the female reproductive system tumor, all neuroendocrine neoplasms were covered in one chapter, low grade neuroendocrine tumor and high grade neuroendocrine carcinoma werenot recommended except for ovary(8). Among 4 types of tumors, typical carcinoid and atypical carcinoid were extremely rare, and SCNEC was by far the most common of these tumours. while So far, only about 70 cases of LCNEC have been reported.(9).In this group of cases,SCNEC was the most common, with twenty cases,otherwise only one case of LCNEC was detected. There were no cases of typical carcinoid and atypical carcinoid tumors. In addition, according to WHO classification standard of lung neuroendocrine tumor, if there were two types of tumors 
components were detected at the same time, and each component was at least more than $10 \%$, it could be diagnosed as admixed with neuroendocrine carcinoma, and the mix components may include squamous cell carcinoma, adenocarcinoma, etc(10). Some invasive squamous cell carcinoma, adenocarcinoma of the cervix and their precusors lesions may coexist with neuroendocrine carcinoma, but adenocarcinoma and glandular precursor lesions seem to be more common. There were 13 cases of pure CNECC and 8 cases of mix types of neuroendocrine cancers. There were 3 cases accompanied by squamous cell cancers, 5 cases accompanied by adenocarcinoma.In pure CNECC, two cases accompanied by HSIL,one case accompanied by In situ adenocarcinoma (AIS).

In this study of CNECC,the patient's average age was 48.6 years old (range:33-69 years). The clinical manifestations of cervical neuroendocrine tumor varied, the presentation of neuroendocrine tumours (NETs) were similiar to other cervical carcinomas, including vaginal bleeding, drainage, and/or detection of a distinct cervical mass(1). The common symptoms in our cohort of patients include irregular vaginal bleeding and drainage, which were similar to other types of cervical carcinomas and lack of specificity.Most of the patients with no neuroendocrine related symptoms, occasionally associated with carcinoid syndrome, etc, but were extremely rare, only a handful of low level neuroendocrine tumor could produce a variety of peptide calcitonin, such as gastric secrete element, serotonin, etc, and in rare cases, ectopic hormone product could cause symptoms or due to metastatic disease later development of carcinoid syndrome.Although most neuroendocrine carcinomas had the potential to differentiate into endocrine cells, they couldnot secrete peptide hormones, or the peptide hormones secreted did not have immunobiological activity and the activity was too low to produce corresponding symptoms(11).In this group, no neuroendocrine symptoms were found.

The pathogenesis of cervical neuroendocrine neoplasms were still unclear. Some cervical low-level neuroendocrine neoplasms were generally secondary to their precancerous lesions - endocrine cell proliferation (12)『Cervical neuroendocrine carcinoma was associated with human papillomavirus infection, High-risk HPV could be detected in most cervical neuroendocrine carcinomas, similar to squamous cell arcinoma(13,14).HPV infection played a critical role in the pathogenesis of cervical neuroendocrine carcinoma(15). In a recent meta analysis, Castle et al(14)analyzed HPV infection data in 403 cases of small cell neuroendocrine carcinomas and 45 cases of large cell neuroendocrine carcinomas. They found that more than $80 \%$ of cases were HPV positive. The predominant subtypes of HPV were HPV 18 and 16. The authors concluded that the E6 protein of HPV binds to the wild-type p53 protein and makes it lose its function, which may be the underlying cause of cervical neuroendocrine carcinoma and that most of cases could be prevented by prophylactic HPV vaccination.In addition, some cervical SCNEC had recurrent genetic alterations involving the MAPK,PI3K/AKT/mTOR, and p53/BRCA pathways(16).Genetic studies have found that, similar with small-cell carcinoma of the lung, small-cell carcinoma of the cervix also had a high frequency of 3p alleles with an occasional 9p21 deletion(1), suggesting that they have some similar cause in the complex process of tumor formation.In this group, HR-HPV tests were performed for 9 cases, 7 cases were positive(7/9, 77.8\%), and 2 cases were negative, specific types did not detect in this study.

CNECC in the early stages only infiltrated stroma tisssues, epithelium tissues were infiltrated rarely, so the conventional cytology screening for cervical neuroendocrine cancer was often not sensitive, specificity was also very poor, the detection rate of neuroendocrine cancer was significantly lower than that of squamous cell carcinoma,the early cervical cytology was often negative.In this study, 9 patients had cervical cytology screening before biopsy, 5 cases were negative, only 4 cases were positive,was diagnosed with HSIL, neuroendocrine carcinoma cells werenot detected in cytological screening.Cytologically, the findings included: A single

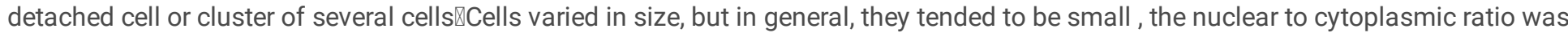
very high.The nuclei were hyperchromatin and coarse-grained, sometimes "squeezed" phenomenon in cells were visible at times. Therefore,HSIL was often diagnosed in some cases,because HSIL cells were very small, the nuclear to cytoplasmic ratio was very high,but the chromatin of the nucleus is very coarse. Another possibility is that cervical cytology only brushed to the epithelial part, neuroendocrine carcinoma can be accompanied by squamous cell carcinoma or HSIL lesions, the cervical squamous epithelial lesions were only detected under the microscope. For the 4 cases of cytology positive, the final pathological diagnosis were 2 cases accompanied by squamous carcinomas, 2 cases accompanied by HSILs, the others 5 cases of cytology negative were not accompanied by other lesions,it wasnot found in cervical cytological screening,because of given priority to with infiltrating stromal elements of pure neuroendocrine cancer cells, only accompanied by others pathological changes at the same time maybe improve the cytology positive detection rate. This group cases falled into the latter category. Therefore, no matter what the TCT results were, increasing the detection of high-risk HPV in patients, especially cervical biopsy in symptomatic patients, could significantly improve the diagnosis rate of early cervical neuroendocrine cancer. Combined examination of TCT and HPV could remarkably improve the detection rate of CNECC before biopsy. In this study, 17 cases were diagnosed as neuroendocrine carcinoma by cervical biopsy and 4 cases werenot diagnosed as $\mathrm{CNECC}$, and they were found in the subsequent radical resected specimens.It may be related to the sampling site of colposcopy is too superfical , too few sampling specimens, and the limited experience of pathologist, etc.It suggested that they 
had some similar cause in the complex process of tumor formation.In this group, HR-HPV tests were performed for 9 cases,7 cases were positive $(7 / 9,77.8 \%)$ by the method of $\mathrm{HC} 2$. and 2 cases were negative, specific types did not detect in this study.

Immunohistochemical staining could provide support for diagnosis of CNECC,and was an important auxiliary differential diagnosis method, on general recognition was one kind of epithelial tissue markers positive (CKpan,EMA, etc.) plus two kinds of neuroendocrine markers (CgA,Syn,CD56, neuronspecific enolase NSE) positive, diagnosis can be made, which of CD56 sensitivity is the most high, the positive rate can reach $80 \%(17)$. But its specificity is very poor, CD56 staining can be present in non-neuroendocrine carcinomas(18). Syn is more sensitive than CgA for CNECC, CgA and PGP9.5 were less so. In our study, Both of Syn and CD56 were positive in 18 cases $(85.7 \%, 18 / 21),(85.7 \%, 18 / 21)$, otherwise, $\mathrm{CgA}$ was positive in 9 cases $(42.9 \%, 9 / 21)$,and the positive staining was more often diffuse than focal. which was similar to previously reported positive rate(19).p16INK4a was diffuse or focally positive in 17 cases $(81 \%, 17 / 21)$, this was similar with To Van report(20).In their study,p16INK4a overexpression was not found to be correlated with p53 or NRAS mutation,suggested that p16INK4a overexpression was significantly correlated with the presence of high-risk HPV strains in neuroendocrine carcinoma of the cervix. Because it may occur in the cervix, p63 can be positive occasionally. In our study,p63 was positive in 9 cases $(42.9 \%, 9 / 21)$. TTF1 was commonly positive $(52.4 \%, 11 / 21)$ and this finding was of no value in the distinction from a pulmonary synonymous primary tuomors $(21,22)$.

Differential diagnosis:CNECC was mainly differentiated from poorly differentiated adenocarcinoma囚squamous cell carcinoma of the cervix and metastatic NECC. Sometimes poorly differentiated carcinomas may be accomplied by focal regional neuroendocrine differentiation, but the proportion should not exceed 10\%. Otherwise, mix CNECC should be considered. Neuroendocrine markers(Syn,CgA,CD56,etc) of poorly differentiated carcinomas may occasionally be isolated neuroendocrine cells positive, they were generally focal positive and rarely diffuse(22). Because CNECC were histologically indistinguishable from others synonymous neuroendocrine tumors, it was important to rule out the possibility metastasis from another site.Sometimes it was necessary to differentiate with lymphoma, endometrial stromal sarcoma, etc. Under the microscope, lymphoma mainly consisted of diffuse round cells with obvious nuclear atypia and no organoid structure. Immunohistochemical markers CD3 or CD20 were commonly positive for leukocyte, while neuroendocrine markers were negative. Endometrial stromal sarcoma tumor cells were usually fusiform or stellate, with abundant or transparent cytoplasm and CD10 was positive,and neuroendocrine markers were negative.

Like their pulmonary counterparts, CNECC were aggressive tumors with high metastatic potential, even were diagnosed at low stage(5).Moreover, because of its rarity, They were frequently misdiagnosed,and there was no standardized treatment regimen, which brings great challenges to clinical diagnosis and treatment. Hence, treatment options have been adapted from management used for neuroendocrine tumors of the lung. Early stage patients ( $\mathbb{\nabla} \rrbracket$ A period) was given priority to with radical surgery and chemotherapy, middle-late patients ( $\nabla \mathrm{B}-\mathbb{\mathrm { B }}$ period) was given priority to with radiation and chemotherapy (23).It mainly covered the comprehensive treatment of operation, radiotherapy and chemotherapy. A multivariate analysis by Embry et al. showed that incorporation of chemotherapy at any point in cancer treatment was associated with prolonged survival. Age, high clinical stage, lymph node metastasis and interstitial infiltration depth were relatively clear independent factors affecting prognosis(24).

In conclusion,CNECC is a extremely rare primary tumor.The tumor is associated with HPV infection. Combined examination of TCT and HPV can significantly improve the detection rate of neuroendocrine carcinoma before biopsy. Pthology diagnosis is based on histological and immunohistochemical examination. It is considered to be highly aggressive malignancy with poor prognosis. Hence, we should prefer to highlight the importance of considering neuroendocrine tumor as differential diagnosis when assessing for potential malignancies at an earlier stage.Further depth research are carried on CNECC from the perspective of molecular biology, so as to seek more targeted treatment methods for clinical practice.

\section{Abbreviations}

CNECC :Neuroendocrine carcinoma of the cervix; HE: hematoxylin-eosinstaining;HPV: human papillomavirus ;HC2: Hybrid Capture 2; TCT:Thinprep cytology test; SCNEC:small cell neuroendocrine carcinoma ; LCNEC:large cell neuroendocrine carcinoma ; HSIL: highgrade squamous intraepithelial lesion;

\section{Declarations}

\section{Acknowledge ments}

We are grateful to the Department of Pathology, Peking University People's Hospital for providing access to archival materials. 
Authors' contributions

XZ initiated the study and wrote the manuscript. DS supervised the research and revised the manuscript. YG carried out immunohistochemistry study. All authors have read and approved the final version of the manuscript.ML have done the follow-up of cases. All authors

have read and approved the final version of the manuscript.

\section{Funding}

Not applicable

\section{Availability of data and material s}

The datasets generated during and/or analyzed for this study are available from the corresponding author upon reasonable request.

\section{Ethics approval and consent to participate}

Ethical approval for the study was obtained from the ethics committee of Peking University People's Hospital (Beijing, China) and the exemption frominformed consent was approved as well.

\section{Consent for publication}

Not applicable.

\section{Competing interests}

The authors declare that they have no competing interests.

\section{Author details}

1 Department of Pathology, Peking University People's Hospital, 11Xizhimen South Street, Xicheng District, Beijing 100044, China. 2 Department of Obstetrics and Gynecology, Peking University People's Hospital, 11Xizhimen South Street, Xicheng District, Beijing 100044, China.

\section{Conflict of interest}

No financial or other potential conflicts of interest exist for any of the authors.

\section{Acknowledgement}

None

\section{References}

1. Kurman RJ, Carcangiu ML, Herrington CS and RH. Y: Who classification of tumours of fe $\neg$ male reproductive organs. . 2014.

2. Gadducci A, Carinelli S and Aletti G: Neuroendrocrine tumors of the uterine cervix: A therapeutic challenge for gynecologic oncologists. Gynecologic Oncology 144: 637-646, 2017.

3. Guadagno E, De Rosa G and Marialaura DBDC: Neuroendocrine tumours in rare sites: differences in nomenclature and diagnostics-a rare and ubiquitous histotype. Journal of Clinical Pathology 69: 563-574, 2016.

4. Hara H, Ishii E, Honda T, Nakagawa M, Teramoto K and Oyama T: Cytological features of atypical carcinoid combined with adenocarcinoma of the uterine cervix. Diagnostic Cytopathology, 2011.

5. Qian Z, Yao X, Ye J, Zhang L, Li L and Wei Q: Influence of clinicopathological characteristics and comprehensive treatment models on the prognosis of small cell carcinoma of the cervix: A systematic review and meta-analysis. Plos One 13: e0192784, 2018. 
6. Reagan JW, Hamonic MJ and Wentz WB: Analytical study of the cells in cervical squamous-cell cancer. Laboratory Investigation 6: 241-250, 1957.

7. Alboressaavedra J, Gersell D, Gilks CB, Henson DE and Al E: Terminology of endocrine tumors of the uterine cervix: results of a workshop sponsored by the College of American Pathologists and the National Cancer Institute. Archives of Pathology \& Laboratory Medicine 121: 34-39, 1997.

8. Board. TWCoTE: Who classification of tumours of fe - male reproductive organs.: 451-459, 2020.

9. Nishio Y, Miyatake T, Yasuoka H, Tsuji H and Tsujimoto M: Primary Neuroendocrine Carcinoma of the Uterine Cervix Treated With Complete Surgical Resection and Adjuvant Combination Chemotherapy. Journal of Clinical Gynecology \& Obstetrics 6: 23-27, 2017.

10. William D.Travis EBAPBea: WHO Classification of Tumours of the Lung,Pleuras, Thymus and Heart.: 66-66, 2015.

11. Wei Bao XW: Progress in the diagnosis and treatment of cervical small cell neuroendocrine carcinoma $\mathbb{J} \rrbracket$. International Journal of Obstetrics and Gynecology, 35: 178-181, 2008.

12. Wang KL, Yang YC, Wang TY, et al.: Neuroendocrine carcinoma of the uterine cervix: A clinicopathologic retrospective study of 31 cases with prognostic implications. Journal of Chemotherapy 18: 209-216, 2006.

13. Wang HL and Lu DW: Detection of human papillomavirus DNA and expression of $p 16, \mathrm{Rb}$, and $\mathrm{p} 53$ proteins in small cell carcinomas of the uterine cervix. American Journal of Surgical Pathology 28: 901-908, 2004.

14. Castle PE, Pierz A and Stoler MH: A systematic review and meta-analysis on the attribution of human papillomavirus (HPV) in neuroendocrine cancers of the cervix. Gynecologic Oncology 148: 422, 2018.

15. Tempfer CB, Tischoff I, Dogan A, et al.: Neuroendocrine carcinoma of the cervix: A systematic review of the literature. Bmc Cancer 18: 530, 2018.

16. Xing D, Zheng G, Schoolmeester JK, et al.: Next-generation Sequencing Reveals Recurrent Somatic Mutations in Small Cell Neuroendocrine Carcinoma of the Uterine Cervix. The American Journal of Surgical Pathology, 2018.

17. Feng Min ZJ, Zhang Yan,Sun Liang, et al.: Neuroendocrine carcinoma of cervix:a clinicopathologic study of 82 cases. . Chinese Journal of Pathology . 47: 328-333, 2018.

18. Emerson RE, Michael H, Wang M, Zhang S and Cheng L: Cervical Carcinomas With Neuroendocrine Differentiation: A Report of 28 Cases With Immunohistochemical Analysis and Molecular Genetic Evidence of Common Clonal Origin With Coexisting Squamous and Adenocarcinomas. International Journal of Gynecological Pathology Official Journal of the International Society of Gynecological Pathologists 35: 1, 2015.

19. Wang Y, Mei K, Xiang MF, Li JM and Xie RM: Clinicopathological characteristics and outcome of patients with small cell neuroendocrine carcinoma of the uterine cervix: case series and literature review. European Journal of Gynaecological Oncology 34: 307-310, 2013.

20. Ta TV, Nguyen QN, Truong VL, Tran TT and Vuong LD: Human Papillomavirus Infection, p16INK4a Expression and Genetic Alterations in Vietnamese Cervical Neuroendocrine Cancer. Malaysian Journal of Medical Sciences 26: 151-157, 2019.

21. Mccluggage WG, Kennedy K and Busam KJ: An immunohistochemical study of cervical neuroendocrine carcinomas:

Neoplasms that are commonly TTF1 positive and which may express CK20 and P63. American Journal of Surgical Pathology 34: 525, 2010.

22. Ono K, Yokota NR, Yoshioka E, et al.: Metastatic large cell neuroendocrine carcinoma of the lung arising from the uterus: A pitfall in lung cancer diagnosis. Pathology - Research and Practice: 654-657, 2016.

23. Frumovitz M: Small- and Large-Cell Neuroendocrine Cervical Cancer. Oncology 30, 2016. 
24. Barni, Sandro, Ghidini, et al.: Second line with oxaliplatin- or irinotecan-based chemotherapy for gemcitabine-pretreated pancreatic cancer: A systematic review. European journal of cancer: official journal for European Organization for Research and Treatment of Cancer (EORTC) European Association for Cancer Research (EACR), 2017.

\section{Figures}

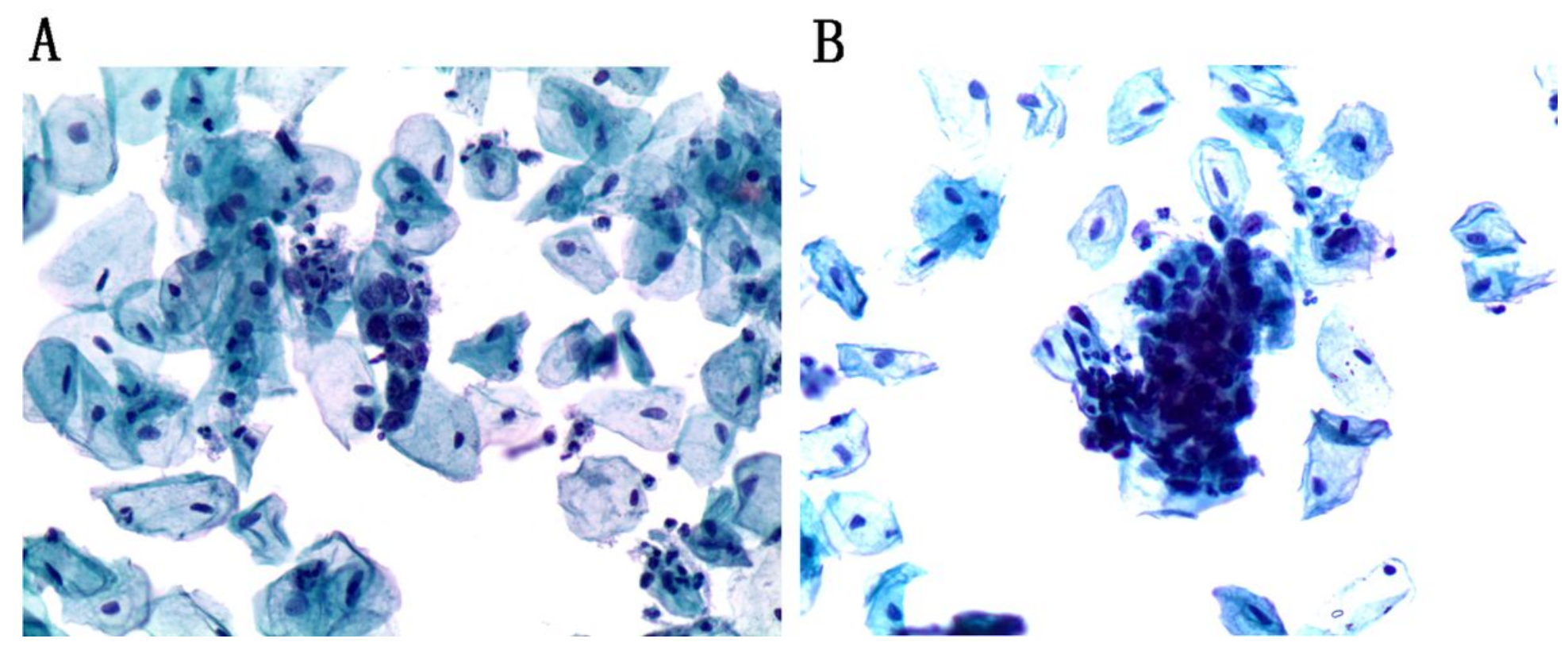

Figure 1

The cytological features of SCNEC accompied with HSIL.(A)The cells display single detached cell or cluster of several cells $邓$ the cells were very small in size, the nuclear to cytoplasmic ratio was very high.The nuclei were hyperchromatin and coarse-grained.(B) The cells display a larger cluster of several cells. 


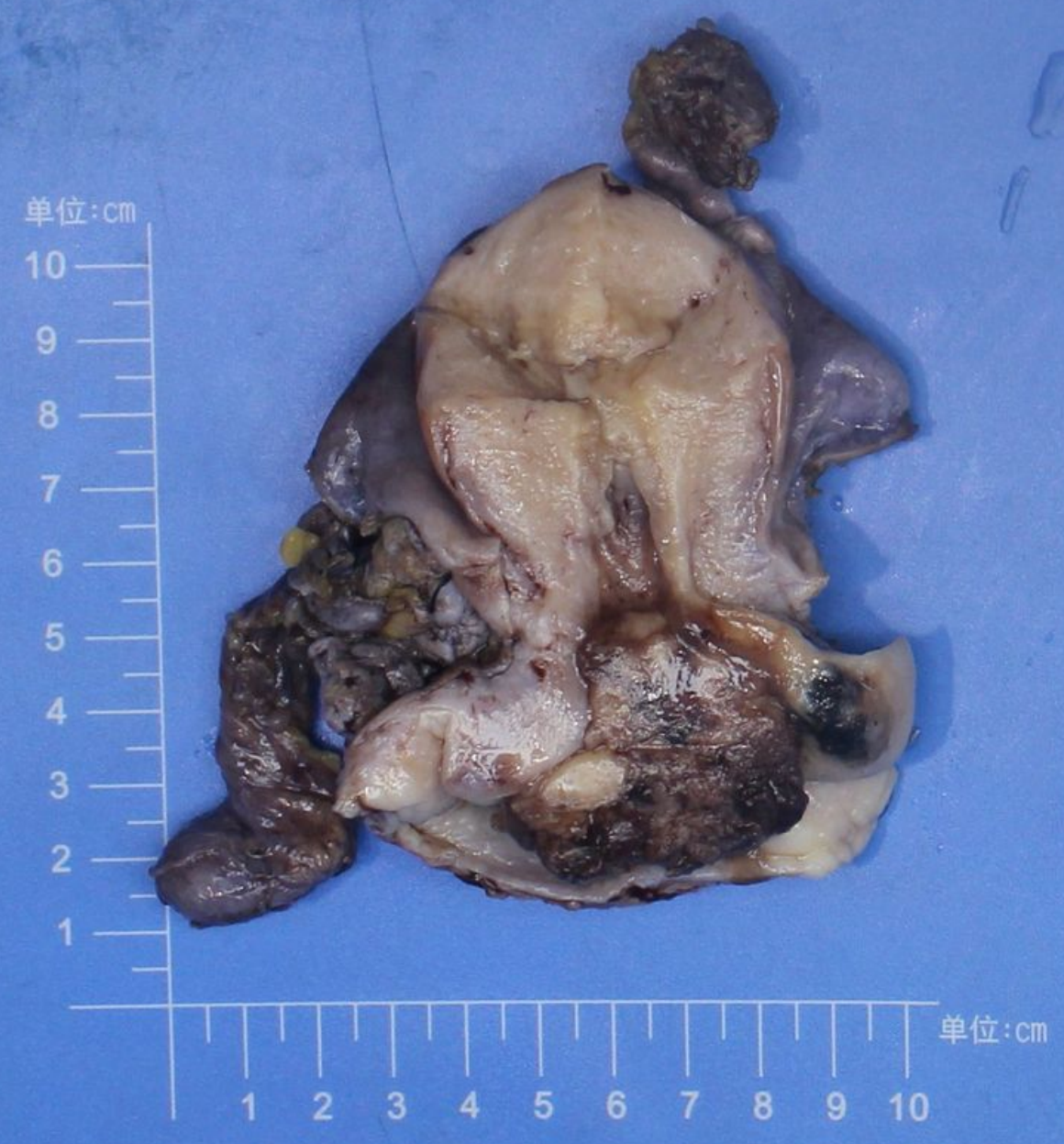

\section{Figure 2}

Representative image of SCNEC,showed exogenous polyps.

A

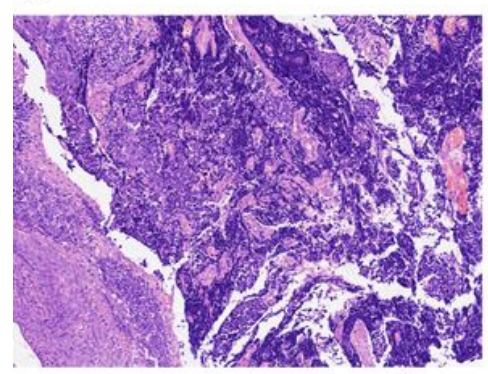

E

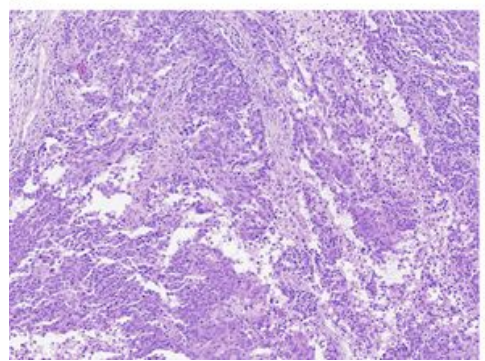

B

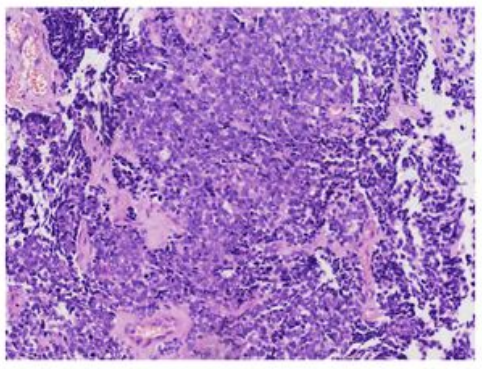

$\mathrm{F}$

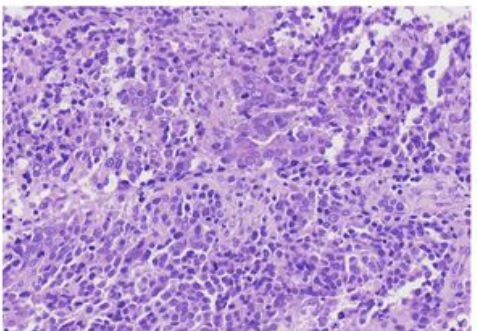

C

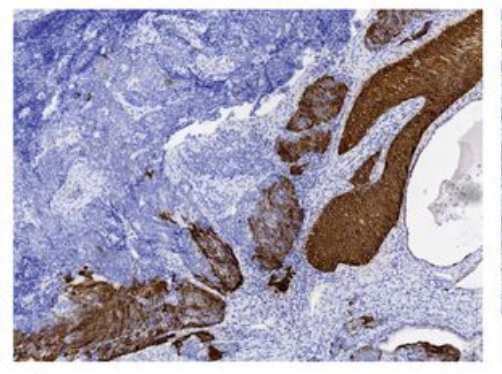

G

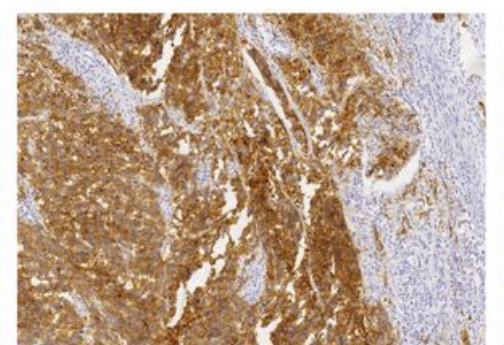

D

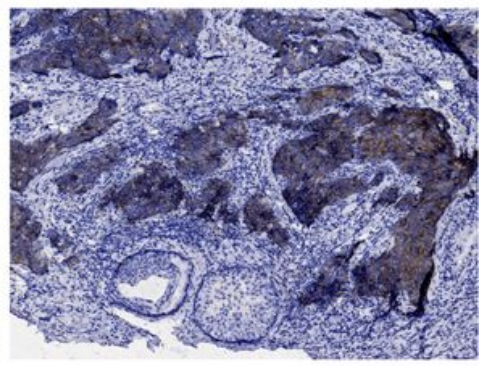

$\mathrm{H}$

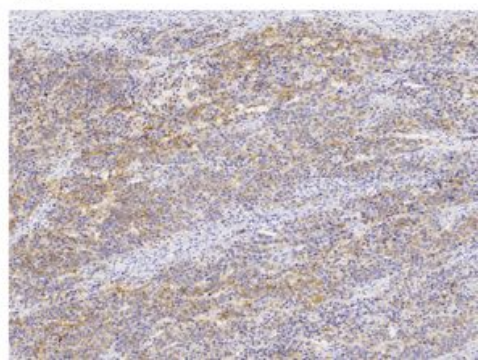




\section{Figure 3}

(A) HE staining of SCNEC showing carcinoma cells arranged in diffuse patches. (B) At high magnification, the cancer cells with hyperchromatic nuclei,scant cytoplasm and abundant mitotic activity,and partly extruded, the nuclear to cytoplasmic ratio was very high. (C) Immunohistochemical staining of CK5/6,SCNEC cells were negative,HSIL cells were positive.(D) Immunohistochemical staining of Syn, SCNEC cells were positive,HSIL cells were negative.(E) HE staining of LCNEC showing carcinoma cells arranged in organ-like structures.(F) At high magnification, cancers cells with prominent nucleoli, abundant cytoplasm.(G) Immunohistochemical staining of Syn positive of LCNEC specimen.(H) Immunohistochemical staining of CgA positive of LCNEC specimen.

\section{Supplementary Files}

This is a list of supplementary files associated with this preprint. Click to download.

- file.pdf 